\title{
DIFFUSION SEMIGROUPS ON ABSTRACT WIENER SPACE
}

\author{
BY \\ M. ANN PIECH( $\left.{ }^{1}\right)$
}

\begin{abstract}
The existence of a semigroup of solution operators associated with a second order infinite dimensional parabolic equation of the form $\partial u / \partial t=L_{x} u$ was previously established by the author. The present paper investigates the relationship between $L_{x}$ and the infinitesimal generator $\mathscr{U}$ of the semigroup. In particular, it is shown that $\mathscr{U}$ is the closure of $L_{x}$ in a natural sense. This in turn implies certain uniqueness results for both the semigroup and for solutions of the parabolic equation.
\end{abstract}

I. Introduction. A pure second order linear differential operator acting on a function whose domain is an open subset of a real separable Hilbert space $H$ is of the form $L_{x} f(x) \equiv \operatorname{trace}\left[A(x) f^{\prime \prime}(x)\right] . A(x)$ is a linear operator on $H$ and $f^{\prime \prime}$ is the second Fréchet derivative of $f$. When $H$ is finite dimensional this coincides with the usual definition, and $L_{x} f(x)$ exists provided $f^{\prime \prime}(x)$ exists. However, when $H$ is infinite dimensional, summability questions arise-for example, whether $A(x) f^{\prime \prime}(x)$ is of trace class. Another dissimilarity to the finite dimensional case is related to the nonavailability of a substitute for Lebesgue measure which is countably additive on $\mathrm{H}$. This is the reason that we work within the context of abstract Wiener space.

In a paper entitled Potential theory on Hilbert space [4], L. Gross has discussed the Laplacian, the related heat equation and the similarities and dissimilarities between finite and infinite dimensional situations. Previous work of the author [7], [9], [10] has centered on extending the results of Gross to second order differential operators with variable coefficients. The present work continues in the same spirit.

The existence of a fundamental solution for $\partial u / \partial t=L_{x} u$ is known under certain smoothness and ellipticity hypotheses on $A(x)$. Associated with this fundamental solution is a semigroup of "solution operators" $\left\{q_{t}: t>0\right\}$. In the finite dimensional case a variety of uniqueness results are available: for the fundamental solution, for the semigroup, and for individual solutions of the differential equation. The proofs of such results have generally not been adaptable to an infinite dimensional situation. Consequently, very little is known concerning uniqueness, even in the case of the Laplacian. This paper was motivated by the desire both for uniqueness

Received by the editors July 14, 1971.

AMS 1970 subject classifications. Primary 47D05, 35K15; Secondary 60J60,28A40.

Key words and phrases. Abstract Wiener space, diffusion semigroups, infinitesimal generator, parabolic equations, Wiener process, uniqueness of semigroup, uniqueness of solutions.

${ }^{1}$ ) This work was supported by NSF contract GP-19651.

Copyright (C) 1972, American Mathematical Society 
results and for a better understanding of the relationship between the infinitesimal generator of $\left\{q_{t}\right\}$ and the differential operator $L_{x}$. It turns out that uniqueness of the semigroup is a consequence of the fact that the infinitesimal generator is the closure of $L_{x}$ in a natural sense.

§II deals with the background and necessary notation. In §III we present the results connecting $L_{x}$ and $\left\{q_{t}\right\}$, including uniqueness results. All smoothness estimates involving $\left\{q_{t}\right\}$ which are necessary for proofs of the results of $\S$ III are deferred until §IV. In §IV we present only such estimates.

II. Background and notation. Let $H$ be a real separable Hilbert space with inner product $(\cdot, \cdot)$ and norm $|\cdot|$. Let $\|\cdot\|$ be a fixed measurable norm on $H$ in the sense of Gross [3] and $B$ be the completion of $H$ with respect to $\|\cdot\|$. The triple $(H, B, i)$ where $i$ is the inclusion mapping of $H$ into $B$ is known as an abstract Wiener space [3]. Denoting by $B^{*}$ and $H^{*}$ the topological dual spaces of $B$ and of $H$ respectively, we may consider $B^{*}$ as being embedded by restriction in $H^{*}$. If then $H^{*}$ is identified with $H$ via the inner product $(\cdot, \cdot)$, we may regard $B^{*}$ as contained in $H$ and hence, via $i$, as contained in $B .\langle\cdot, \cdot\rangle$ will denote the natural pairing of $B^{*}$ and $B$. For each $t>0, p_{t}$ will denote the Wiener measure on the Borel field of $B$ which is determined by Gauss cylinder set measure $v_{t}$, with variance parameter $t$, on $H$ [3]. For $x$ in $B$ and for each Borel set $\Gamma$ in $B, p_{t}(x, \Gamma) \equiv p_{t}(\Gamma-x)$.

We will deal with two notions of differentiability of a function $f$ from $B$ to some Banach space $W . f$ will be called $B$-differentiable at $x$ if $f$ is Fréchet differentiable at $x$. This derivative will be denoted by $D f(x)$ and it is a member of the space $L(B, W)$ of bounded linear maps from $B$ to $W$. We also may consider the behavior of $f$ only at points of the form $x+h$ with $h$ in $H$. Then we will say that $f$ is $H$ differentiable at $x$ if there exists an $f^{\prime}(x)$ in $L(H, W)$ satisfying

$$
\left|f(x+h)-f(x)-f^{\prime}(x) h\right|_{w}=o(|h|)
$$

for small $h$ in $H$. Since the $H$ norm is stronger than the $B$ norm, $B$-differentiability of $f$ at $x$ implies $H$-differentiability of $f$ at $x$. We will write $\|D f(x)\|$ and $\left|f^{\prime}(x)\right|$ for the $L(B, W)$ and $L(H, W)$ norms respectively.

Let $L_{x}$ be a second order differential operator of the form

$$
L_{x} f(x) \equiv \operatorname{trace}\left[A(x) f^{\prime \prime}(x)\right]
$$

where $A(\cdot)$ is a map from $B$ to $L(H)(\equiv L(H, H))$, and $f$ is a real-valued function on $B$. We will denote by $L_{x, t}$ the differential operator

$$
L_{x, t} u(x, t) \equiv \operatorname{trace}\left[A(x) u^{\prime \prime}(x, t)\right]-(\partial / \partial t) u(x, t)
$$

where $u$ is a real-valued function on $B \times(0, \infty)$ and ' denotes $H$-differentiability, for $t$ fixed. We say that $L_{x} f$ or $L_{x, t} u$ exists if the relevant derivatives exist and if $A(x) f^{\prime \prime}(x)$ or $A(x) u^{\prime \prime}(x, t)$, respectively, is trace class in $L(H)$. Under certain hypotheses on $L_{x}$ concerning ellipticity and smoothness, it is established in [7] that a 
fundamental solution for $L_{x, t} u(x, t)=0$ exists. Explicitly, if we assume that $A(x)$ is of the form $I-B(x)$, where

(i) $B(x)$ is a symmetric member of $L(H)$ and there exists an $\varepsilon>0$ such that $B(x) \leqq(1-\varepsilon) I$ for all $x$ in $B$;

(ii) there exists a symmetric Hilbert-Schmidt operator $E$ on $H$ such that $B(x)$ is of the form $E B_{0}(x) E$, where $B_{0}(x) \in L(H)$ and $\left|B_{0}(x)\right|_{L(H)} \leqq 1$ for all $x$ in $B$;

(iii) $D^{2} B_{0}(x)$ exists and is a bounded Lip-1 function from $B$ to

$$
L(B \rightarrow L(B \rightarrow L(H))) ;
$$

(iv) $\left\|D B_{0}(\cdot)\right\|$ is uniformly bounded;

(v) there exists a constant $c$ such that for any orthonormal basis $\left\{e_{i}\right\}$ of $H$ we have $\sum_{i}\left|D B_{0}(x) e_{i}\right|_{L(H)}^{2}<c$, independently of $x$ in $B$;

then there exists a family of finite real-valued signed Borel measures $\left\{q_{t}(x, d y): 0<t<\infty, x \in B\right\}$ on $B$ such that if

$$
q_{t} f(x) \equiv \int_{B} f(y) q_{t}(x, d y)
$$

then, for each bounded real-valued Lip-1 function $f$ on $B, L_{x, t} q t f(x)=0$ for all $x$ in $B$ and $t>0$. Moreover, $\left\|q_{t} f-f\right\|_{\infty} \rightarrow 0$ as $t \downarrow 0$.

Henceforth we will assume that $A(x)$ satisfies the preceding conditions (i)-(v). With a few additional hypotheses of a technical nature, it is shown in [9] that $q_{t}(x, d y)$ is a probability measure on $B$ and $q_{t} q_{s} f(x)=q_{t+s} f(x)$ for each bounded real-valued measurable function $f$ on $B$, for all $s$ and $t$ greater than 0 and for all $x$ in $B$.

It has recently been announced by Kuo and Piech [6] in the presence of the additional hypotheses

(vi) there exists an increasing sequence $\left\{P_{n}\right\}$ of finite dimensional projections on $B$ such that $P_{n}[B] \subset B^{*},\left\{P_{n}\right\}$ converges strongly to the identity on $B$, and $\left\{P_{n} / H\right\}$ converges strongly to the identity on $H$;

(vii) $B(\cdot): B \rightarrow L\left(B, B^{*}\right)$;

that $\left\{q_{t}(x, d y)\right\}$ is a family of probability measures satisfying the aforementioned semigroup property. Henceforth we will assume that (vi) and (vii) hold.

The following outlines the approach of [6]. Let $\Omega$ denote the space of continuous functions $\omega$ from $[0, \infty)$ into $B$ and vanishing at $0 . \mathscr{M}$ is the $\sigma$-field of $\Omega$ generated by the functions $\omega \rightarrow \omega(t) . \mathscr{P}$ is that unique probability measure on $\mathscr{M}$ for which the condition $0=t_{0}<t_{1}<\cdots<t_{n}$ implies that $\omega\left(t_{j+1}\right)-\omega\left(t_{j}\right), 0 \leqq j \leqq n-1$, are independent and $\omega\left(t_{j+1}\right)-\omega\left(t_{j}\right)$ has distribution measure $p_{t_{j+1}-t_{j}}$ in $B$. The process $W_{t}$ defined by $W_{t}(\omega) \equiv \omega(t)$ is called a Wiener process on $B$. Regarding $A(x)$ as a member of $L(B)$, which is made possible by (vii), it is shown that the stochastic integral equation

$$
X_{t}(\omega)=X_{0}(\omega)+\int_{0}^{t}\left[A\left(X_{s}(\omega)\right)\right]^{1 / 2} d W_{s}(\omega)
$$


has a unique solution $X_{t}$ which is a homogeneous strong Markov process. Defining

$$
\hat{q}_{t / 2}(x, d y) \equiv \mathscr{P}\left\{X_{t} \in d y: X_{0}=x\right\},
$$

it is asserted in [6] that $q_{t}(x, d y)=\hat{q}_{t}(x, d y)$ for all $t>0$ and $x$ in $B$. For the proof of this theorem, it is necessary to verify the following: $\left(^{*}\right)$ For each bounded real-valued Lip-1 function $f$ on $B$, the maps $(x, t) \rightarrow\left(q_{t} f\right)^{\prime}(x)$ from $B \times(0, \infty)$ to $H$ with $|\cdot|$ and $(x, t) \rightarrow\left(q_{t} f\right)^{\prime \prime}(x)$ from $B \times(0, \infty)$ to the space of trace class operators on $H$ with trace class norm are continuous.

Since [6] is an announcement and does not contain a proof of $\left({ }^{*}\right)$, we will make these estimates as part of §IV.

As a consequence of the equality of $q_{t}$ and $\hat{q}_{t}$, we have the following uniqueness result:

TheORem [6]. Assume $L_{x}$ and B satisfy (i)-(vii). Then the family $\left\{q_{t}(x, d y): t>0\right.$, $x \in B\}$ whose existence is established in [7] is unique among families $\left\{\mu_{t}(x, d y): t>0\right.$, $x \in B\}$ of bounded real-valued signed Borel measures on $B$ which satisfy the following requirements:

For each bounded real-valued Lip-1 function fon $B$, setting $\mu_{t} f(x) \equiv \int_{B} f(y) \mu_{t}(x, d y)$,

(a) $\mu_{t} f(x)$ satisfies $L_{x, t} \mu_{t} f(x)=0$,

(b) $\left\|\mu_{t} f-f\right\|_{\infty} \rightarrow 0$ as $t \downarrow 0$,

(c) $(x, t) \rightarrow\left(\mu_{t} f\right)^{\prime}(x)$ and $(x, t) \rightarrow\left(\mu_{t} f\right)^{\prime \prime}(x)$ are continuous from $B \times(0, \infty)$ to $H$ and to the space of trace class operators on $H$ respectively.

We will denote by $\mathscr{L}$ the Banach space of bounded uniformly continuous realvalued functions on $B$ with the sup norm $\|\cdot\|_{\infty} . \mathscr{A}$ will denote the Banach space of Lip-1 functions $f$ in $\mathscr{L}$, with norm

$$
\|f\|_{1}=\|f\|_{\infty}+\inf \{c:|f(x)-f(y)| \leqq c\|x-y\| \text { for all } x \text { and } y \text { in } B\} .
$$

It is established in [10, Theorem 4] that $\left\{q_{t}: t>0\right\}$ forms a strongly continuous contraction semigroup on $\mathscr{L}$. We remark that the results of [10], while presupposing that the $q_{t}(x, d y)$ are probability measures and satisfy the semigroup property, are independent of the additional hypotheses made in [9]. Let $\mathscr{U}$ denote the infinitesimal generator of the semigroup $\left\{q_{t}\right\}$ on $\mathscr{L}$. We define $\mathscr{S}$ as the set of all $f$ in $\mathscr{L}$ such that $f^{\prime}$ is continuous as a map from $B$ to $H$ and $f^{\prime \prime}$ is bounded and uniformly continuous as a map from $B$ to trace class operators on $H$. $L_{x} f$ is thus defined for all $f$ in $\mathscr{S}$. In $\S$ III we establish that $\mathscr{S}$ is in the domain of $\mathscr{U}$. This proof utilizes the representation of $\left\{q_{t}(x, d y)\right\}$ as the family of transition probabilities of the solution of the stochastic integral equation (1), and thus relies on the smoothness properties $\left(^{*}\right)$ which we establish in $§ I V$. The proof also utilizes Kuo's generalization of a formula of $\mathrm{K}$. Itô to stochastic integrals on abstract Wiener space [5, Theorem 4.1]. We are eventually able to show that $\left\{q_{t}\right\}$ is the unique contraction semigroup on $\mathscr{L}$ whose infinitesimal generator extends $\left(L_{x}, \mathscr{S}\right)$. 
The facts from basic semigroup theory which we will use in the next section may all be found in Dynkin [1, Chapter 1]. In particular, we now quote two such theorems for future reference.

TheOREM [1, THEOREM 1.1]. Let $T_{t}$ be a contraction semigroup on a Banach space $V$ and let $\mathcal{O}$ be its infinitesimal generator. Let $V_{0} \equiv\left\{g\right.$ in $V:\left\|T_{t} g-g\right\|_{V} \rightarrow 0$ as $t \downarrow 0\}$. Then for arbitrary $g$ in $V_{0}$ the equation $\lambda f-O f=g(\lambda>0)$ has one and only one solution $f$ in the domain $\mathscr{D}_{\mathcal{O}}$ of $\mathcal{O}$. This solution is given by the formula

$$
f=\int_{0}^{\infty} e^{-\lambda t} T_{t} g d t
$$

Moreover $\|f\|_{V} \leqq(1 / \lambda)\|g\|_{V}$.

THEOREM [1, THEOREM 1.2]. Let $T_{t}$ and $T_{t}^{\prime}$ be two contraction semigroups on a Banach space $V$. If their infinitesimal generators coincide, then $T_{t} g=T_{t}^{\prime} g$ for all $g$ in $V_{0}$. Here $V_{0}$ does not depend on which of the two given semigroups determines it.

III. The relationship of $\mathscr{U}$ and $L_{x}$. We begin the study of the relationship between $\mathscr{U}$ and $L_{x}$ by proving that if $f$ and its first two $H$-derivatives are reasonably smooth then $f$ is in the domain of $\mathscr{U}$ and $\mathscr{U}$ coincides with $L_{x}$ on $f$. Recall that the domain of $\mathscr{U}$ is defined as the set of all $f$ in $\mathscr{L}$ which satisfy $\left\|\left(q_{t} f-f\right) / t\right\|_{\infty}$ converges as $t \downarrow 0 . \mathscr{U} f$ is defined to be the limit of $\left(q_{t} f-f\right) / t$.

TheOREM 1. $\mathscr{S}$ is in the domain of $\mathscr{U}$. For $f$ in $\mathscr{S}, \mathscr{U} f=L_{x} f$.

Proof. Fix $x$ in $B$ and $f$ in $\mathscr{S}$. Let $X_{t}$ be the unique solution of the stochastic integral equation

$$
X_{t}(\omega)=x+\int_{0}^{t}\left[A\left(X_{s}(\omega)\right)\right]^{1 / 2} d W_{s}(\omega)
$$

Then $q_{t / 2}(x, d y)=\mathscr{P}\left\{X_{t} \in d y\right\}$. Applying Itô's formula [5, Theorem 4.1],

$$
f\left(X_{t}\right)-f(x)=\int_{0}^{t}\left\langle\left[A\left(X_{s}\right)\right]^{1 / 2} f^{\prime}\left(X_{s}\right), d W_{s}\right\rangle+\int_{0}^{t} \frac{1}{2} \operatorname{trace}\left[A\left(X_{s}\right) f^{\prime \prime}\left(X_{s}\right)\right] d s .
$$

Letting $\mathscr{E}$ denote expectation with respect to $\mathscr{P}$, it follows from $[5,(4)$ of Theorem 3.4] that

$$
\mathscr{E} \int_{0}^{t}\left\langle\left[A\left(X_{s}\right)\right]^{1 / 2} f^{\prime}\left(X_{s}\right), d W_{s}\right\rangle=0 .
$$

Moreover $\mathscr{E}\left(f\left(X_{t}\right)\right)=q_{t / 2} f(x)$ and

$$
\mathscr{E} \int_{0}^{t} \frac{1}{2} \operatorname{trace}\left[A\left(X_{s}\right) f^{\prime \prime}\left(X_{s}\right)\right] d s=\int_{0}^{t} q_{s / 2}\left[\frac{1}{2} \operatorname{trace} A(x) f^{\prime \prime}(x)\right] d s .
$$

Setting $g(x)=\frac{1}{2}$ trace $A(x) f^{\prime \prime}(x)$, we take expectations on each side of equation (2), obtaining

$$
q_{t ; 2} f(x)-f(x)=\int_{0}^{t} q_{s / 2} g(x) d s .
$$


Thus

$$
\begin{aligned}
\left\|\frac{q_{t / 2} f-f}{t / 2}-2 g\right\|_{\infty} & =\frac{2}{t}\left\|\int_{0}^{t}\left(q_{s / 2} g-g\right) d s\right\|_{\infty} \leqq \frac{2}{t} \int_{0}^{t}\left\|q_{s / 2} g-g\right\|_{\infty} d s \\
& \leqq 2 \sup _{0<s \leqq t}\left\|q_{s / 2} g-g\right\|_{\infty} \rightarrow 0 \text { as } t \downarrow 0
\end{aligned}
$$

since $g \in \mathscr{L}$ and $\left\{q_{t}\right\}$ is strongly continuous on $\mathscr{L}$. This shows that $\mathscr{U} f=2 g=L_{x} f$, as desired.

REMARK. The choice of $\mathscr{S}$ was determined by the following considerations. $\mathscr{S}$ must naturally be a subset of $\mathscr{L}$ since we are considering the semigroup $\left\{q_{t}\right\}$ as acting in $\mathscr{L} . f^{\prime}$ and $f^{\prime \prime}$ must have sufficient smoothness to enable use in equation (2) of Itô's formula-that is, $f^{\prime}$ must be continuous from $B$ to $H$ and $f^{\prime \prime}$ must be continuous from $B$ into the space of trace class operators on $H$. Finally, we used the requirement that $q_{t}\left(\right.$ trace $\left.A(x) f^{\prime \prime}(x)\right)$ be strongly continuous at $t=0$. This is achieved by requiring $L_{x} f(x)$ to be in $\mathscr{L}$. Since $A(\cdot)$ and $A(\cdot)^{-1}$ are bounded and uniformly continuous from $B$ to $L(H), L_{x} f(x)$ is in $\mathscr{L}$ if $f^{\prime \prime}(\cdot)$ is bounded and uniformly continuous from $B$ to the trace class operators on $H$.

Let us regard the differential operator $L_{x}$ as having domain of definition the subset $\mathscr{S}$ of $\mathscr{L}$, and thus as having range in $\mathscr{L}$. From basic semigroup theory, $\mathscr{U}$ is a closed operator. That is, $\{(f, \mathscr{U} f): f$ is in the domain of $\mathscr{U}\}$ is closed in $\mathscr{L} \times \mathscr{L}$. The next theorem shows that $\mathscr{U}$ is equal to the closure of $\left(L_{x}, \mathscr{S}\right)$.

THEOREM 2. $\mathscr{S}$ is dense in the domain of $\mathscr{U}$ in the graph norm.

The proof of Theorem 2 is provided by the following two lemmas. Recall that $\mathscr{A}$ consists of the Lip-1 functions in $\mathscr{L}$.

LEMMA 2.1. For $f$ in $\mathscr{L}$, let

$$
Q f(x) \equiv \int_{0}^{\infty} e^{-t}\left(q_{t} f\right)(x) d t
$$

Then $f \in \mathscr{A} \Rightarrow Q f \in \mathscr{S}$.

The proof of Lemma 2.1 will be provided by the smoothness estimates which we will make in §IV.

LEMma 2.2. $\{Q f: f \in \mathscr{A}\}$ is dense in the domain of $\mathscr{U}$ with respect to the graph norm.

Proof. Assume $u$ is in the domain of $\mathscr{U}$. Set $g \equiv(I-\mathscr{U}) u$. Then it is a consequence of the strong continuity of $q_{t}$ that

$$
u=(I-\mathscr{U})^{-1} g=\int_{0}^{\infty} e^{-t}\left(q_{t} g\right) d t
$$

By a lemma of Gross [4, Lemma 3.2.1], $\mathscr{A}$ is dense in $\mathscr{L}$ with respect to the sup norm. Thus, given $\varepsilon>0$, we may find $f \in \mathscr{A}$ such that $\|g-f\|_{\infty}<\varepsilon$. Set

$$
v \equiv(I-\mathscr{U})^{-1} f=Q f \text {. }
$$


Since $\left\|(I-\mathscr{U})^{-1}\right\| \leqq 1$, we have $\|u-v\|_{\infty}<\varepsilon$ and

$$
\begin{aligned}
\|\mathscr{U} u-\mathscr{U} v\|_{\infty} & =\|(I-\mathscr{U}-I)(u-v)\|_{\infty} \leqq\|(I-\mathscr{U})(u-v)\|_{\infty}+\|u-v\|_{\infty} \\
& =\|g-f\|_{\infty}+\|u-v\|_{\infty}<2 \varepsilon .
\end{aligned}
$$

The question now naturally arises of whether any larger closed extension of $\left(L_{x}, \mathscr{S}\right)$ can exist and generate a contraction semigroup on $\mathscr{L}$. Or, putting this question in a different and less rigorous form, is the family of solution operators $\left\{q_{t}\right\}$ associated with $L_{x, t} u=0$ unique? This is answered by the following theorem, whose proof is a simple consequence of basic semigroup theory coupled with the fact of strong continuity of $\left\{q_{t}\right\}$ on $\mathscr{L}$.

THEOREM 3. If $\left\{q_{t}^{\prime}: t>0\right\}$ is a contraction semigroup on $\mathscr{L}$ whose infinitesimal generator $\mathscr{U}^{\prime}$ extends $\left(L_{x}, \mathscr{S}\right)$, then $q_{t}^{\prime}=q_{t}$ for all $t>0$.

Proof. We need only show that the domain of $\mathscr{U}^{\prime}$ coincides with the domain of $\mathscr{U}$ and that $\mathscr{U}^{\prime}=\mathscr{U}$ on their common domain. Since $\left(\mathscr{U}^{\prime}, \mathscr{D}_{\mathscr{U}^{\prime}}\right)$ must be closed and since $\left(\mathscr{U}, \mathscr{D}_{\mathscr{U}}\right)$ is the closure of $\left(L_{x}, \mathscr{S}\right)$, we automatically have $\left(\mathscr{U}^{\prime}, \mathscr{D}_{\mathscr{U}}\right) \supset\left(\mathscr{U}, \mathscr{D}_{\mathscr{U}}\right)$. Suppose now that $f \in \mathscr{D}_{\mathscr{U}^{\prime}}$. Set $g=f-\mathscr{U}^{\prime} f$. Define $\mathscr{L}_{0} \equiv\left\{h\right.$ in $\mathscr{L}:\left\|q_{t}^{\prime} h-h\right\|_{\infty} \rightarrow 0$ as $t \downarrow 0\}$. Since $\mathscr{L}_{0} \supset \mathscr{D}_{\mathscr{U}}$, and $\mathscr{U}^{\prime}: \mathscr{L}_{0} \rightarrow \mathscr{L}_{0}$, we have $g \in \mathscr{L}_{0}$. Thus $f$ is the unique member of $\mathscr{D}_{\mathscr{U}}$, satisfying $f-\mathscr{U}^{\prime} f=g$. Since $q_{t}$ is strongly continuous on $\mathscr{L}$, there exists a unique $\tilde{f}$ in $\mathscr{D}_{\mathscr{U}}$ such that $\tilde{f}-\mathscr{U} \tilde{f}=g$. But since $\tilde{f} \in \mathscr{D}_{\mathscr{U}}, \mathscr{U}^{\prime} \tilde{f}=\mathscr{U} \tilde{f}$ and so $\tilde{f}=f$. Thus $\left(\mathscr{U}^{\prime}, \mathscr{D}_{\mathscr{U}^{\prime}}\right) \subset\left(\mathscr{U}, \mathscr{D}_{\mathscr{U}}\right)$.

REMARK. The preceding theorem may also be proved by application of the corollary to Theorem 1.1 of [1].

Uniqueness of individual solutions of $L_{x, t} u=0$ has not yet been studied. Theorem 1 provides us with a rather large set $(\mathscr{S})$ of specific functions in the domain of $\mathscr{U}$ and tells us how $\mathscr{U}$ acts on these functions. Theorem 1.3 of [1] now immediately gives the following uniqueness result.

THEOREM 4. For $f$ in $\mathscr{S}$, the function $u(t, x) \equiv q_{t} f(x)$ is the unique solution of $L_{x, t} u$ $=0$ which satisfies the conditions:

(a) $u(t, \cdot)$ is Borel measurable,

(b) $\partial u / \partial t$ exists uniformly $\left(\|\cdot\|_{\infty}\right)$ and is uniformly continuous for $t \geqq 0$,

(c) $\|u(t, \cdot)\|_{\infty} \leqq c_{1} \exp \left(c_{2} t\right)$ where $c_{1}$ and $c_{2}$ are some constants, and

(d) $\|u(t, \cdot)-f(\cdot)\|_{\infty} \rightarrow 0$ as $t \downarrow 0$.

IV. Smoothness of $q_{t} f(x)$. For a Borel set $\Gamma$ in $B$, equation (32) of [7] shows that $q_{t}(x, \Gamma)$ is of the form

$$
q_{t}(x, \Gamma)=\hat{m}_{t}(x, \Gamma)+\int_{0}^{t} \int_{B} r_{u}(y, \Gamma) \hat{m}_{t-u}(x, d y) d u,
$$

where $\left\{\hat{m}_{t}(x, d y): t>0, x \in B\right\}$ and $\left\{r_{t}(x, d y): t>0, x \in B\right\}$ are families of finite real-valued signed Borel measures on $B$. We will employ the notation

$$
\hat{m}_{t} f(x) \equiv \int_{B} f(y) \hat{m}_{t}(x, d y), \quad r_{t} f(x) \equiv \int_{B} f(y) r_{t}(x, d y) .
$$


The desired smoothness properties will first be established for $\hat{m}_{t} f(x)$, together with some estimates which will enable us to carry limits through the time integral which occurs in the expression for $q_{t} f(x)$. Smoothness in the case where $q_{t}(x, d y)$ $=p_{t}(x, d y)$ has been established in Theorem 3 of [4]. Estimates occurring therein are fundamental to the calculations which follow.

For $f$ bounded, real-valued Borel measurable on $B$,

$$
\hat{m}_{t} f(x) \equiv \int_{B}[\operatorname{det} A(y)]^{-1 / 2} f(y) e^{-(C(x)(x-y), x-y) / 4 t} p_{2 t}(x, d y),
$$

with $C(x) \equiv A(x)^{-1}-I$. The exponential term is to be interpreted as that measurable function on $B$ determined by the function $y \rightarrow(C(x)(x-y), x-y)$, which is defined on $H+x$ and uniformly continuous near zero in the topology generated by the measurable seminorms on $H+x$ (see [7], equations (5) and (6)). Define $\hat{f}(y)$ $\equiv$ [det $A(y)]^{-1 / 2} f(y) . \hat{f}$ is bounded and Borel measurable (uniformly continuous, Lip-1, respectively) if and only if $f$ is. Moreover, $f \rightarrow\|f\|_{\infty}$ and $f \rightarrow\|\hat{f}\|_{\infty}$ are equivalent norms on $\mathscr{L} ; f \rightarrow\|f\|_{1}$ and $f \rightarrow\|\hat{f}\|_{1}$ are equivalent norms on $\mathscr{A}$. Letting

$$
m_{t} f(x) \equiv \int_{B} f(x+y) e^{-(C(x) y, y) / 4 t} p_{2 t}(d y),
$$

we observe that the change of variables $y \rightarrow y-x$ gives $m_{t} \hat{f}(x)=\hat{m}_{t} f(x)$. Consequently, we will make all our estimates for $m_{t} f(x)$.

The measure $m_{t}(x, d y)$ may be interpreted in terms of Wiener measure on a suitable abstract Wiener space. For $h$ and $k$ in $H$ and $x$ in $B$, define $(h, k)_{x}$ $\equiv\left(A(x)^{-1 / 2} h, A(x)^{-1 / 2} k\right)$. Let $H^{x}$ be the vector space $H$ with inner product $(\cdot, \cdot)_{x}$. The topologies given by $(\cdot, \cdot)$ and $(\cdot, \cdot)_{x}$ are equivalent, and so we may also regard $B$ as the completion of $H^{x}$ with respect to $\|\cdot\|$. It is then a consequence of Theorem 3 of [10] that we may interpret

$$
p_{t}^{x}(d y) \equiv[\operatorname{det} A(x)]^{-1 / 2} e^{-(C(x) y, y) / 2 t} p_{t}(d y)
$$

as that Wiener measure on $B$ which is induced by Gauss measure on $H^{x}$ with variance parameter $t$. Thus we have

$$
m_{t} f(x)=[\operatorname{det} A(x)]^{1 / 2} p_{2 t}^{x} f(x) .
$$

In this form, $m_{t} f(x)$ has a three-fold dependence on $x$. The change in the right side of (7) caused by a change in either of the first two occurrences of $x$ is influenced by the properties of the coefficients $A(\cdot)$; whereas the effect of a change in only the third " $x$ " is independent of the $A(\cdot)$, and is determined by the behavior of the fundamental solution of the Laplacian.

LEMMA 5.1. There exist constants $\tau>1$ and $c$ such that

$$
\int_{B}|f(y)| p_{1}^{x}(d y) \leqq c\left\{\int_{B}|f(y)|^{\tau} p_{1}(d y)\right\}^{1 / \tau}
$$

for all $x$ in $B$, provided $f \in L^{\tau}\left(p_{1}\right)$. 
Proof. It is shown in [7, p. 99] that $e^{-(C(x) y, y) / 2} \in L^{1+\lambda}\left(p_{1}(d y)\right)$ for all positive $\lambda$ which are sufficiently close to zero. For such a $\lambda$, the $L^{1+\lambda}$ norm is uniformly bounded with respect to $x$. If $\tau^{-1}+\lambda^{-1}=1$, then the lemma follows upon application of Hölder's inequality.

By Proposition 1 of [7], for fixed $\tau>0$ the map $x \rightarrow\left(m_{t} f\right)(x)$ is twice $H$ differentiable at each $x$ in $B$. The derivatives are given by

$$
\left(\left(m_{t} f\right)^{\prime}(x), h\right)
$$

$$
=(4 t)^{-1} \int_{B} f(x+y)\left[-\left(C^{\prime}(x) h y, y\right)+2\left(A(x)^{-1} h, y\right)\right] e^{-(C(x) y, y) / 4 t} p_{2 t}(d y),
$$

and

$\left(\left(m_{t} f\right)^{\prime \prime}(x) k, h\right)$

$$
=-(4 t)^{-1} \int_{B} f(x+y)\left\{\left[\left(C^{\prime \prime}(x) k h y, y\right)-2\left(C^{\prime}(x) h k y, y\right)\right.\right.
$$

$$
\begin{aligned}
& \left.-2\left(C^{\prime}(x) k h y, y\right)+2\left(A(x)^{-1} h, k\right)\right] \\
& -\left[\left(C^{\prime}(x) h y, y\right)-2\left(A(x)^{-1} h, y\right)\right] \cdot(4 t)^{-1} \\
& \left.\cdot\left[\left(\bar{C}^{\prime}(x) k y, y\right)-2\left(A(x)^{-1} k, y\right)\right]\right\} e^{-(C(x) y, y) / 4 t} p_{2 t}(d y),
\end{aligned}
$$

valid for all $h$ and $k$ in $H$. Making the change of variables $y \rightarrow(2 t)^{1 / 2} y$, we observe that, for fixed $h$ and $k$ in $H$,

$$
t \rightarrow m_{t} f(x), \quad t \rightarrow\left(\left(m_{t} f\right)^{\prime}(x), h\right), \quad t \rightarrow\left(\left(m_{t} f\right)^{\prime \prime}(x) k, h\right)
$$

are Borel measurable. If in addition $f$ is Lip-1, these three functions are uniformly continuous on intervals of the form $0<a \leqq t \leqq b<\infty$.

Since $H$ is separable, the weak measurability of $\left(m_{t} f\right)^{\prime}(x)$ implies its strong $t$ measurability. Moreover, we may estimate the $H$ norm of $\left(m_{t} f\right)^{\prime}(x)$ as follows:

$$
\begin{aligned}
&\left|\left(m_{t} f\right)^{\prime}(x)\right|_{H}=\sup _{h \in H:|h|=1}\left|\left(\left(m_{t} f\right)^{\prime}(x), h\right)\right| \\
& \leqq c\|f\|_{\infty} \sup _{h \in H ;|h|=1}\left\{\int_{B}\left[\left|\left(C^{\prime}(x) h y, y\right)\right|+t^{-1 / 2}\left|\left(A(x)^{-1} h, y\right)\right|\right] e^{-(C(x) y, y) / 2} p_{1}(d y)\right\} \\
& \leqq c\|f\|_{\infty} \sup _{h \in H ;|h|=1}\left\{\int_{B}|h||F y|^{2} e^{-(C(x) y, y) / 2} p_{1}(d y)\right. \\
&\left.+t^{-1 / 2} \int_{B}\left|\left(A(x)^{-1} h, y\right)\right| e^{-(C(x) y, y) / 2} p_{1}(d y)\right\} .
\end{aligned}
$$

$c$ always denotes a general constant; it will in general depend on the coefficients $A(\cdot)$ and on the abstract Wiener space $B$ which are fixed in this paper, but will be independent of all variables in our calculations. $F$ is a symmetric Hilbert-Schmidt operator on $H$ such that $C(x), C^{\prime}(x)(\cdot)$ and $C^{\prime \prime}(x)(\cdot)(\cdot)$ are of the form $F C_{0}(x) F$, $F C_{1}(x)(\cdot) F$ and $F C_{2}(x)(\cdot)(\cdot) F$ respectively. The existence and properties of these operators are proved in [7]. $|F y|^{2}$ is in $L^{p}\left(p_{1}\right)$ for all $p \geqq 1$; thus Lemma 5.1 gives 
the existence of a constant $c$ such that

$$
\int_{B}|F y|^{2} e^{-(C(x) y, y) / 2} p_{1}(d y) \leqq c
$$

for all $x$ in $B$. Upon making the "change of variable" $y \rightarrow A(x)^{1 / 2} y$, which may be justified by Theorem 3 of [11], we obtain

$$
\begin{aligned}
\int_{B}\left|\left(A(x)^{-1} h, y\right)\right| e^{-(C(x) y, y) / 2} p_{1}(d y) & \\
& \leqq c \int_{B}\left|\left(A(x)^{-1 / 2} h, y\right)\right| p_{1}(d y) \leqq c\left|A(x)^{-1 / 2} h\right| \leqq c|h|
\end{aligned}
$$

for all $x$ in $B$. Thus we conclude that

$$
\left|\left(m_{t} f\right)^{\prime}(x)\right| \leqq c\|f\|_{\infty}\left(1+t^{-1 / 2}\right)
$$

for all $x$ in $B, t>0$ and for all bounded measurable $f$.

Writing

$$
\begin{aligned}
& \left(\left(m_{t} f\right)^{\prime}(x), h\right) \\
& \quad=\int_{B} f\left(x+(2 t)^{1 / 2} y\right)\left[-\left(C^{\prime}(x) h y, y\right) / 2+(2 t)^{-1 / 2}\left(A(x)^{-1} h, y\right)\right] e^{-(C(x) y, y) / 2} p_{1}(d y),
\end{aligned}
$$

calculations analogous to the preceding show that if $f$ is in $\mathscr{A}$ then

$$
\left|\left(m_{t} f\right)^{\prime}(x)-\left(m_{s} f\right)^{\prime}(x)\right|_{H} \leqq c\|f\|_{1}\left[\left|t^{1 / 2}-s^{1 / 2}\right|+\left|t^{-1 / 2}-s^{-1 / 2}\right|\right] .
$$

Thus $t \rightarrow\left(m_{t} f\right)^{\prime}(x)$ is Lip-1 on intervals of the form $0<a \leqq t \leqq b<\infty$, uniformly for all $x$ in $B$.

To establish $x$-smoothness of $\left(m_{t} f\right)^{\prime}(x)$ we assume that $f$ is in $\mathscr{A} . x \rightarrow|A(x)|$ and $x \rightarrow \operatorname{det} A(x)$ are uniformly bounded above and away from zero, and are in Lip-1 (see [7]). From [7, p. 94] we infer the existence of an $x_{3}$ in $B$ such that

$$
\begin{aligned}
\left|\left(\left[C^{\prime}\left(x_{1}\right)-C^{\prime}\left(x_{2}\right)\right] h y, y\right)\right| & =\left|\left(D^{2} C\left(x_{3}\right)\left(x_{1}-x_{2}\right) h y, y\right)\right| \\
& =\left|\left(F C_{2}\left(x_{3}\right)\left(x_{1}-x_{2}\right) h F y, y\right)\right| \\
& \leqq c\left\|x_{1}-x_{2}\right\| \cdot|h| \cdot|F y|^{2} .
\end{aligned}
$$

If $\beta$ is assigned a fixed value $\geqq 1$ and sufficiently close to 1 , calculations in [7, $\mathrm{p}$. 109] show that

$$
\left\{\int_{B}\left|\exp \left(-\left(C\left(x_{1}\right) y, y\right) / 2\right)-\exp \left(-\left(C\left(x_{2}\right) y, y\right) / 2\right)\right|^{\beta} p_{1}(d y)\right\}^{1 / \beta} \leqq c\left\|x_{1}-x_{2}\right\| .
$$

Thus, changing variables $y \rightarrow(2 t)^{1 / 2} y$ in equation (8), applications of Lemma 5.1 and of Hölder's inequality easily yield

$$
\left|\left(m_{t} f\right)^{\prime}\left(x_{1}\right)-\left(m_{t} f\right)^{\prime}\left(x_{2}\right)\right| \leqq c\|f\|_{1} \cdot\left\|x_{1}-x_{2}\right\|\left(1+t^{-1 / 2}\right) .
$$

We may now conclude that the family $\left\{F_{f}:\|f\|_{1} \leqq 1\right\}$ where $F_{f}(x, t) \equiv\left(\hat{m}_{t} f\right)^{\prime}(x)$ is uniformly equicontinuous as a family of functions from $B \times(0, \infty)$ to $H$. 
We turn now to consideration of $\left(m_{t} f\right)^{\prime \prime}(x)$. In order that $\left(m_{t} f\right)^{\prime \prime}(x)$ be of trace class, we will assume that $f$ is in $\mathscr{A}$. We may write

$$
\left(m_{t} f\right)^{\prime \prime}(x)=[\operatorname{det} A(x)]^{1 / 2}\left[V_{t}(x)+W_{t}(x)+X_{t}(x)\right],
$$

where for all $h$ and $k$ in $H$,

$$
\begin{aligned}
&\left(V_{t}(x) k, h\right) \equiv(2 t)^{-1} \int_{B} f(x+y)\left[-(h, k)_{x}+(h, y)_{x}(k, y)_{x} / 2 t\right] p_{2 t}^{x}(d y) \\
&\left(W_{t}(x) k, h\right) \equiv \int_{B} f(x+y)\{-(4 t)^{-1}\left(C^{\prime \prime}(x) k h y, y\right) \\
&+(2 t)^{-1}\left[\left(C^{\prime}(x) h k, y\right)+\left(C^{\prime}(x) k h, y\right)\right] \\
&\left.+\left(16 t^{2}\right)^{-1}\left(C^{\prime}(x) h y, y\right)\left(C^{\prime}(x) k y, y\right)\right\} p_{2 t}^{x}(d y)
\end{aligned}
$$

and

$$
\begin{aligned}
\left(X_{t}(x) k, h\right)=\int_{B} f(x+y)\left(-8 t^{2}\right)^{-1}\left[(h, y)_{x}\left(C^{\prime}(x) k y, y\right)\right. & \\
& \left.+(k, y)_{x}\left(C^{\prime}(x) h y, y\right)\right] p_{2 t}^{x}(d y) .
\end{aligned}
$$

We recall that the family of trace class operators in $L(H)$ is

$$
\left\{T \in L(H): \sum_{i=1}^{\infty}\left(\left[T^{*} T\right]^{1 / 2} e_{i}, e_{i}\right)<\infty \text { for some orthonormal basis }\left\{e_{i}\right\} \text { of } H\right\},
$$

with the trace of $T$ defined as $\operatorname{Tr} T \equiv \sum_{i=1}^{\infty}\left(T e_{i}, e_{i}\right)$ where $\left\{e_{i}\right\}$ is any orthonormal basis of $H$. The trace class norm of $T$ is $|T|_{\operatorname{Tr}} \equiv \operatorname{Tr}\left[T^{*} T\right]^{1 / 2}$. The completely continuous operators in $L(H)$ with $|\cdot|_{L(H)}$ form the dual of the Banach space of trace class operators under the pairing $\langle U, V\rangle=\operatorname{Tr} U^{*} V$, where $U$ is completely continuous and $V$ is of trace class. Since operators of finite rank are dense in the space of completely continuous operators, we may write

$$
|T|_{\operatorname{Tr}}=\sup \left\{|\operatorname{Tr}[T F]| /|F|_{L(H)}: F \text { is of finite rank in } L(H) \text { and } F \not \equiv 0\right\} .
$$

For any $S$ in $L(H)$ and $T$ of trace class, $S T$ and $T S$ are of trace class and $\operatorname{Tr} S T$ $=\operatorname{Tr} T S$. Thus the set of trace class operators on $H$ is invariant under a change of inner product. Consequently, the set of trace class operators and their traces are the same whether we consider $L(H)$ or any $L\left(H^{x}\right)$. However, the trace class norm varies with a change of inner product. In our case, there exist constants $c_{1}$ and $c_{2}$ independent of $x$ in $B$ such that

$$
|T|_{L\left(H^{x}\right)} \leqq c_{1}|T|_{L(H)} \leqq c_{2}|T|_{L\left(H^{x}\right)}
$$

Thus there are constants $k_{1}$ and $k_{2}$, also independent of $x$ in $B$, such that

$$
|T|_{\operatorname{Tr} H^{x}} \leqq k_{1}|T|_{\operatorname{Tr} H} \leqq k_{2}|T|_{\operatorname{Tr} H^{x}} .
$$

$|T|_{\operatorname{Tr} H^{x}}$ denotes the trace class norm in $L\left(H^{x}\right)$. We will henceforth write $|T|$ or $|T|_{\operatorname{Tr}}$ instead of $|T|_{L(H)}$ or $|T|_{\operatorname{Tr} H}$, respectively, whenever no confusion may arise. 
$\left(V_{t}(x) k, h\right)=\left(A(x) V_{t}(x) k, h\right)_{x}$. Noting the form of the right side of equation (13), we may now apply Theorem 3 of [4] to conclude that $A(x) V_{t}(x)$ is of trace class. Equation (38) of [4] gives the estimate

$$
\left|A(x) V_{t}(x)\right|_{\operatorname{Tr} H^{x}} \leqq c\|f\|_{1} t^{-1 / 2} \int_{B}\|y\| p_{1}(d y) .
$$

Thus $V_{t}(x)$ is of trace class, and there exists a constant $c$ such that

$$
\left|V_{t}(x)\right|_{\operatorname{Tr}} \leqq c\|f\|_{1} t^{-1 / 2}
$$

for all $t>0$ and for all $x$ in $B$.

Estimates found on pp. 107-108 of [6] are easily seen to imply that, for any operator $T$ of finite rank in $L(H)$,

$$
\left|\operatorname{Tr} T W_{t}(x)\right| \leqq c|T|\|f\|_{\infty} t^{-1 / 2} .
$$

We conclude that $W_{t}(x)$ is of trace class, with

$$
\left|W_{t}(x)\right|_{\operatorname{Tr}} \leqq c\|f\|_{\infty} t^{-1 / 2} .
$$

Let $T$ now be a symmetric linear operator on $H$ of finite rank. Let $\left\{e_{i}: i=1, \ldots\right.$, $n\}$ be any orthonormal (with respect to $(\cdot, \cdot)$ ) basis for the range of $T$ and let $P$ be the orthogonal projection of $H$ onto the span of $\left\{e_{i}: i=1, \ldots, n\right\}$. On replacing $y$ by $(2 t)^{1 / 2} y$, we have

$$
\begin{aligned}
\operatorname{Tr} T X_{t}(x)=c t^{-1 / 2} \int_{B} f(x+y) \sum_{i=1}^{n}\left[\left(T e_{i}, y\right)_{x}\left(C^{\prime}(x) e_{i} y, y\right)\right. \\
\left.+\left(e_{i}, y\right)_{x}\left(C^{\prime}(x) T e_{i} y, y\right)\right] p_{1}^{x}(d y) .
\end{aligned}
$$

For each $y$ in $H$,

$$
\begin{aligned}
\sum_{i=1}^{n}\left[\left(T e_{i}, y\right)_{x}\left(C^{\prime}(x) e_{i} y, y\right)+\left(e_{i}, y\right)_{x}\left(C^{\prime}(x) T e_{i} y, y\right)\right] \\
=\left(C^{\prime}(x)\left[\sum_{i=1}^{n}\left(e_{i}, T A(x)^{-1} y\right) e_{i}+T \sum_{i=1}^{n}\left(e_{i}, A(x)^{-1} y\right) e_{i}\right] y, y\right) \\
=\left(C^{\prime}(x)\left[P T A(x)^{-1} y+T P A(x)^{-1} y\right] y, y\right)
\end{aligned}
$$

and

$\left|\left(C^{\prime}(x)\left[P T A(x)^{-1} y+T P A(x)^{-1} y\right] y, y\right)\right| \leqq c\left[\left\|P T A(x)^{-1} y\right\|_{B}+\left\|T P A(x)^{-1} y\right\|_{B}\right]|E y|^{2}$.

Theorem 5 of [2] gives the following result: for any $S$ in $L(H),\|S y\|$ is a measurable seminorm and for any $\lambda>0$,

$$
\operatorname{prob}\left(\|S y\|^{\sim}>|S|_{L(H)} \lambda\right) \leqq \operatorname{prob}(\|y\|>\lambda),
$$

where prob may be with respect to Wiener measure of arbitrary variance. Thus for any finite $p \geqq 1$,

$$
\|\| S y\|\sim\|_{L^{p}} \leqq|S|_{L(H)}\|\| y\|\|_{L^{p} .}
$$


Applying this result to $P T A(x)^{-1}$ and $T P A(x)^{-1}$; noting that $f$ is bounded, $\left|P T A(x)^{-1}\right|_{L(H)}$ and $\left|T P A(x)^{-1}\right|_{L(H)}$ are $\leqq c|T|_{L(H)}$, and that $|E y|^{\sim}$ is in $L^{p}\left(p_{1}\right)$ for any $p \geqq 1$; and then applying Lemma 5.1 give

$$
\left|\operatorname{Tr} T X_{t}(x)\right| \leqq c\|f\|_{\infty}|T|_{L(H)} t^{-1 / 2} .
$$

Since $X_{t}(x)$ is symmetric, we now may conclude that $X_{t}(x)$ is of trace class and that

$$
\left|X_{t}(x)\right|_{\operatorname{Tr}} \leqq c\|f\|_{\infty} t^{-1 / 2} .
$$

Equations (16), (17) and (18) now show that, for $f$ in $\mathscr{A},\left(m_{t} f\right)^{\prime \prime}(x)$ is of trace class, with

$$
\left|\left(m_{t} f\right)^{\prime \prime}(x)\right|_{\operatorname{Tr}} \leqq c\|f\|_{1} t^{-1 / 2} .
$$

Strong $t$-measurability of $\left(m_{t} f\right)^{\prime \prime}(x)$ follows since the space of trace class operators on $H$ is separable. If we make the change of variables $y \rightarrow(2 t)^{1 / 2} y$ in equations (14) and (15) for $W_{t}(x)$ and $X_{t}(x)$, and note the resulting manner in which $t$ occurs, then the preceding calculations easily imply that the family $\left\{F_{x, f}: x \in B,\|f\|_{1} \leqq 1\right\}$ where $F_{x, f}(t) \equiv W_{t}(x)+X_{t}(x)$ is uniformly equicontinuous as a family of functions from an interval of the form $\{t \geqq a>0\}$ to the space of trace class operators on $H$. In addition, smoothness of $C(x)$ and its derivatives combines with the preceding techniques in a straightforward fashion to yield the inequality

$$
\left|W_{t}\left(x_{1}\right)+X_{t}\left(x_{1}\right)-W_{t}\left(x_{2}\right)-X_{t}\left(x_{2}\right)\right|_{\operatorname{Tr}} \leqq c\|f\|_{1} t^{-1 / 2}\left\|x_{1}-x_{2}\right\| .
$$

Similar statements concerning $V_{t}(x)$ are rather more difficult to prove. We now turn our attention to $V_{t}(x)$.

A test operator $S$ is a bounded linear operator of finite rank from $B$ to $B^{*}$. With $S$ we may associate operators $T$ in $L(H)$ and $T^{x}$ in $L\left(H^{x}\right)$ as follows: Consider the diagrams

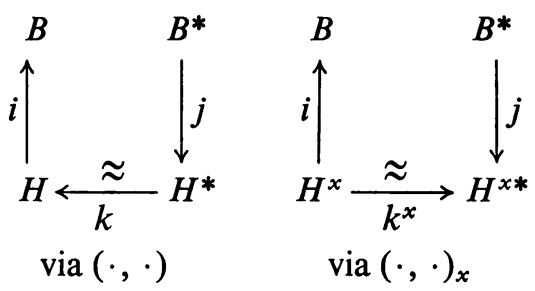

with $i$ the injection of $H$ or $H^{x}$ into $B$ and $j$ the embedding by restriction of $B^{*}$ into $H^{*}$ or $H^{x *}$. $i$ and $j$ do not depend on the inner product assigned to $H$. However, the identification $k$ (or $k^{x}$ ) of $H$ with $H^{*}$ (or of $H^{x}$ with $H^{x *}$ respectively) via the Riesz Representation Theorem does depend on the inner product. For $y$ in $H$ we define $T y=k j$ Siy and $T^{x} y=k^{x} j$ Siy. Since $T^{x}$ (or $T$ ) is a Hilbert-Schmidt operator in $L\left(H^{x}\right)$ (or $L(H)$ respectively), it is trivial to verify that there exists a constant $c$ such that

$$
\left|T^{x}\right|_{\mathrm{H}-\mathrm{S} H^{x}}=|A(x) T|_{\mathrm{H}-\mathrm{S} H^{x}} \leqq c|T|_{\mathrm{H}-\mathrm{S} H}
$$

for all $x$ in $B$. 
For any test operator $S$, Proposition 9 of [4] yields the formula

(21) $\operatorname{Tr}\left[T^{x} A(x) V_{t}(x)\right]=(2 t)^{-1} \int_{B} f(x+y)\left[(2 t)^{-1}\langle S y, y\rangle-\operatorname{Tr} T^{x}\right] p_{2 t}^{x}(d y)$.

Since $\operatorname{Tr}\left[T^{x} A(x) V_{t}(x)\right]=\operatorname{Tr}\left[T A(x) V_{t}(x) A(x)\right]$, we may write

$$
\operatorname{Tr} T\left[A\left(x_{1}\right) V_{t}\left(x_{1}\right) A\left(x_{1}\right)-A\left(x_{2}\right) V_{t}\left(x_{2}\right) A\left(x_{2}\right)\right]=(\mathrm{i})+(\mathrm{ii})+(\mathrm{iii}),
$$

where

$$
\begin{aligned}
& \text { (i) } \equiv(2 t)^{-1} \int_{B}\left[f\left(x_{1}+y\right)-f\left(x_{2}+y\right)\right]\left[(2 t)^{-1}\langle S y, y\rangle-\operatorname{Tr} T^{x_{1}}\right] p_{2 t}^{x_{1}}(d y), \\
& \text { (ii) } \equiv(2 t)^{-1} \int_{B} f\left(x_{2}+y\right)\left[(2 t)^{-1}\langle S y, y\rangle-\operatorname{Tr} T^{x_{1}}\right]\left[p_{2 t}^{x_{1}}(d y)-p_{2 t}^{x_{2}}(d y)\right] \\
& \text { (iii) } \equiv(2 t)^{-1} \int_{B} f\left(x_{2}+y\right)\left[\operatorname{Tr} T^{x_{2}}-\operatorname{Tr} T^{x_{1}}\right] p_{2 t}^{x_{2}}(d y) .
\end{aligned}
$$

We have

$$
\begin{aligned}
|(\mathrm{i})| \leqq & c t^{-1}\left\{\int_{B}\left|f\left(x_{1}+y\right)-f\left(x_{2}+y\right)\right|^{2} p_{2 t}^{x_{1}}(d y)\right\}^{1 / 2} \\
& \cdot\left\{\int_{B}\left|(2 t)^{-1}\langle S y, y\rangle-\operatorname{Tr} T^{x_{1}}\right|^{2} p_{2 t}^{x_{1}}(d y)\right\}^{1 / 2} \\
\leqq & c t^{-1}\|f\|_{1}\left\|x_{1}-x_{2}\right\|\left|T^{x_{1}}\right|_{\mathrm{H}-\mathrm{S} H^{x}} \\
\leqq & c t^{-1}\|f\|_{1}\left\|x_{1}-x_{2}\right\||T|_{\mathrm{H}-\mathrm{S} H},
\end{aligned}
$$

and

$$
\begin{aligned}
&|(\mathrm{ii})| \leqq c\|f\|_{1} t^{-1}\left\{\int_{B}\left|(2 t)^{-1}\langle S y, y\rangle-\operatorname{Tr} T^{x_{1}}\right|^{\alpha} p_{2 t}(d y)\right\}^{1 / \alpha} \\
& \cdot\left\{\int_{B} \mid\left[\operatorname{det} A\left(x_{1}\right)\right]^{-1 / 2} e^{-\left(C\left(x_{1}\right) y, y\right) / 4 t}\right. \\
&\left.-\left[\operatorname{det} A\left(x_{2}\right)\right]^{-1 / 2} e^{-\left(C\left(x_{2}\right) y, y\right) / 4 t} \mid{ }^{\beta} p_{2 t}(d y)\right\}^{1 / \beta}
\end{aligned}
$$

where $\alpha^{-1}+\beta^{-1}=1$. Calculations in [7, p. 109] show that the second integral in the right side of (23) is dominated by a constant times $\left\|x_{1}-x_{2}\right\|$, independently of $t>0$, provided $\beta$ is assigned a fixed value sufficiently close to 1 .

Since equality of $(T h, k)$ and $(T k, h)$ for all $h$ and $k$ in $H$ is equivalent to equality of $\left(T^{x} h, k\right)_{x}$ and $\left(T^{x} k, h\right)_{x}$ for all $h$ and $k$ in $H$, we see that $T$ is symmetric in $L(H)$ if and only if $T^{x}$ is symmetric in $L\left(H^{x}\right)$. If the restriction of $S$ to $L(H)$ is symmetric, then the calculations of $[4, \mathrm{pp} .159-160]$ show that, for fixed $\alpha>1$,

$$
\left\{\int_{B}\left|(2 t)^{-1}\langle S y, y\rangle-\operatorname{Tr} T^{x}\right|{ }^{\alpha} p_{2 t}^{x}(d y)\right\}^{1 / 2} \leqq c_{2}\left|T^{x}\right|_{\mathrm{H}-\mathrm{S} H^{x}}
$$


where $c_{\alpha}$ is a constant depending on $\alpha$ but independent of $x$ in $B$. Since the H-S $H^{x}$ norm of the $L\left(H^{x}\right)$-symmetric part of $T^{x}$ is dominated by a constant times the $\mathrm{H}-\mathrm{S} H^{x}$ norm of $T^{x}$ (the constant being independent of $x$ ), and since $\langle S y, y\rangle$ and the trace of $T^{x}$ depend only on the symmetric part of $T^{x},(23)$ holds for any test operator $S$.

Writing

$$
p_{2 t}(d y)=[\operatorname{det} A(x)]^{1 / 2} e^{(C(x) y, y) / 4 t} p_{2 t}^{x}(d y),
$$

we may easily verify that $[\operatorname{det} A(x)]^{1 / 2} e^{(C(x) y, y) / 4 t}$ is in $L^{\lambda}\left(p_{2 t}^{x}\right)$ for all $\lambda$ in $(1,1+\varepsilon)$ where $\varepsilon>0$ is sufficiently small. The $L^{\lambda}$ norm is uniformly bounded as $x$ varies over $B$. An application of Hölder's inequality to the first integral on the right side of (23) now yields

$$
|(\mathrm{ii})| \leqq c\|f\|_{1} t^{-1}\left\|x_{1}-x_{2}\right\||T|_{\mathrm{H}-\mathrm{S} H} \text {. }
$$

We have

$$
\mid(\text { iii) }) \leqq c\|f\|_{1} t^{-1}\left|\operatorname{Tr}\left[A\left(x_{2}\right)-A\left(x_{1}\right)\right] T\right| \leqq c\|f\|_{1} t^{-1}|T|_{\operatorname{Tr} H} .
$$

For all $S$ of fixed finite rank $n,|T|_{\mathrm{H}-\mathrm{S} H}$ and $|T|_{\operatorname{Tr} H}$ are equivalent norms. Thus

$$
\mid\left(\text { iii) }\left.\left|\leqq c_{n}\|f\|_{1} t^{-1}\left\|x_{1}-x_{2}\right\|\right| T\right|_{\mathrm{H}-\mathrm{S} H} .\right.
$$

Making the identifications $B^{*} \subset H^{*} \approx H$, let $\left\{e_{i}: i=1,2, \ldots\right\}$ be an orthonormal basis of $H$ such that each $e_{i}$ is in $B^{*}$, and let $P_{n}$ denote the orthogonal projection $(H)$ onto the span of $\left\{e_{1}, \ldots, e_{n}\right\}$. Then equations (22), (25) and (26) show that $x \rightarrow P_{n} A(x) V_{t}(x) A(x)$ is in Lip-1 when considered as a map from $B$ to HilbertSchmidt operators with the H-S $H$ norm. Moreover,

$$
\left|P_{n} A\left(x_{1}\right) V_{t}\left(x_{1}\right) A\left(x_{1}\right)-P_{n} A\left(x_{2}\right) V_{t}\left(x_{2}\right)\right|_{\text {н-S } H} \leqq c_{n}\|f\|_{1} t^{-1}\left\|x_{1}-x_{2}\right\|
$$

with $c_{n}$ depending only on the rank $n$. Since in the space of bounded operators on $H$ with range in $P_{n} H$ the H-S $H$ norm and Tr $H$ norm are equivalent, (27) holds with $\mathrm{H}-\mathrm{S} H$ replaced by $\mathrm{Tr}$.

We next wish to show that $P_{n} A(x) V_{t}(x) A(x)$ converges to $A(x) V_{t}(x) A(x)$ as $n$ $\rightarrow \infty$ in $\operatorname{Tr} H$ norm, with the convergence being uniform for all $t \geqq a>0,\|f\|_{1} \leqq 1$ and $x$ in $B$. We view $P_{n}$ as mapping $H$ to $B^{*}$ and let $Q_{n}$ be the unique continuous extension of $P_{n}$ to $L\left(B, B^{*}\right)$. Then $Q_{n}$ is a test operator. We will also view $Q_{n}$ as an operator in $L(B, B)$ via the identifications $B^{*} \subset H^{*} \approx H \subset B$, where the isomorphism of $H^{*}$ and $H$ is given by the inner product $(\cdot, \cdot)$. Then for any test operator $S$, $S\left(Q_{n}-Q_{m}\right)$ is also a test operator. If $T$ and $T^{x}$ are the operators in $L(H)$ and $L\left(H^{x}\right)$ respectively associated with $S$, then $T\left(P_{n}-P_{m}\right)$ and $T^{x}\left(P_{n}-P_{m}\right)$ are the operators in $L(H)$ and $L\left(H^{x}\right)$ associated with $S\left(Q_{n}-Q_{m}\right)$. Now we may interpret equation (35) of [4] as

$$
\left|\operatorname{Tr}\left[T^{x}\left(P_{n}-P_{m}\right) A(x) V_{t}(x)\right]\right| \leqq c\|f\|_{1} t^{-1} \int_{B}\left\|S\left(Q_{n}-Q_{m}\right) y\right\| p_{2 t}^{x}(d y) .
$$


We make the change of variables $y \rightarrow(2 t)^{1 / 2} y$ and apply Lemma 5.1 in order to clarify the time dependence and remove the $x$ dependence of the right side of (28), obtaining

(29) $\left|\operatorname{Tr}\left[T^{x}\left(P_{n}-P_{m}\right) A(x) V_{t}(x)\right]\right| \leqq c\|f\|_{1} t^{-1 / 2}\left\{\int_{B}\left\|S\left(Q_{n}-Q_{m}\right) y\right\|^{\tau} p_{1}(d y)\right\}^{1 / \tau}$

with $\tau$ a fixed constant $>1$. $\left\|S\left(Q_{n}-Q_{m}\right) y\right\|$ is a tame function based on the finite dimensional subspace $M \equiv\left(P_{n}-P_{m}\right) H$, and so we may write

$$
\int_{B}\left\|S\left(Q_{n}-Q_{m}\right) y\right\|^{\tau} p_{1}(d y)=\int_{M}\|T y\|^{\tau} \mu_{1}(d y) .
$$

$\mu_{1}$ is Gauss measure on $(M,(\cdot, \cdot))$ with variance parameter 1 . The calculations on p. 172 of [4] yield the inequality

$$
\int_{M}\|T y\|^{\tau} \mu_{1}(d y) \leqq|T|_{L(H)}^{\tau} \int_{M}\|y\|^{\tau} \mu_{1}(d y)
$$

But

$$
\int_{M}\|y\|^{\tau} \mu_{1}(d y)=\int_{M}\left\|\left(P_{n}-P_{m}\right) y\right\|^{\tau} \mu_{1}(d y)=\int_{B}\left\|\left(Q_{n}-Q_{m}\right) y\right\|^{\tau} p_{1}(d y)
$$

Operators $T^{x}$ of the form which we are considering are dense in the completely continuous operators in $L(H)$, and so equations (28) through (32) give

$$
\left|\left(P_{n}-P_{m}\right) A(x) V_{t}(x)\right|_{\operatorname{Tr} H} \leqq c\|f\|_{1} t^{-1 / 2}\left\{\int_{B}\left\|\left(Q_{n}-Q_{m}\right) y\right\|^{\tau} p_{1}(d y)\right\}^{1 / \tau} .
$$

By Corollary 5.2 of [2], $\left\|\left(Q_{n}-Q_{m}\right) y\right\| \rightarrow 0$ in probability with respect to $p_{1}$. Define

$$
\phi_{n, m}(\lambda) \equiv p_{1}\left(\left\{y:\left\|\left(Q_{n}-Q_{m}\right) y\right\|^{\tau}>\lambda\right\}\right) .
$$

As $n, m \rightarrow \infty$ we have $\phi_{n, m}(\lambda) \rightarrow 0$ for each $\lambda>0$. Define

$$
\phi(\lambda) \equiv p_{1}\left(\left\{y:\|y\|^{\imath}>\lambda\right\}\right)
$$

When $n \neq m,\left|P_{n}-P_{m}\right|_{L(H)}=1$, and so by Theorem 5 of [2] $\phi_{n, m}(\lambda) \leqq \phi(\lambda)$. (For $n=m$ this inequality is trivially valid.) Thus

$$
\int_{B}\left\|\left(Q_{n}-Q_{m}\right) y\right\|{ }^{\tau} p_{1}(d y)=-\int_{0}^{\infty} \lambda d \phi_{n, m}(\lambda)
$$

$\lambda \phi(\lambda)=o(1)$ as $\lambda \rightarrow \infty$, since $\lambda \phi(\lambda) \leqq \int_{\|y\| \geq \lambda 1 / \tau}\|y\|^{\tau} p_{1}(d y)$ and $\|y\|^{\tau} \in L^{1}\left(p_{1}\right)$. "Integrating by parts" gives

$$
\int_{B}\left\|\left(Q_{n}-Q_{m}\right) y\right\|^{x} p_{1}(d y)=\int_{0}^{\infty} \phi_{n, m}(\lambda) d \lambda .
$$


Since $\int_{0}^{\infty} \phi(\lambda) d \lambda=\int_{B}\|y\|^{\tau} p_{1}(d y)<\infty$ it follows by dominated convergence that

$$
\int_{0}^{\infty} \phi_{n, m}(\lambda) d \lambda \rightarrow 0 \text { as } m, n \rightarrow \infty \text {. }
$$

(33), (34) and (35) imply our desired result: $P_{n} A(x) V_{t}(x) A(x)$ converges to $A(x) V_{t}(x) A(x)$ in Tr norm, the convergence being uniform for all $t \geqq a>0,\|f\|_{1} \leqq 1$ and $x$ in $B$. Now the uniform continuity of $P_{n} A(x) V_{t}(x) A(x)$ (equation (27)) and of $A(x)^{-1}$ together with their uniform boundedness (see equation (16)) imply that the family $\left\{F_{t, f}: t \geqq a>0\right.$ and $\left.\|f\|_{1} \leqq 1\right\}$ with $F_{t, f}(x) \equiv V_{t}(x)$ is uniformly equicontinuous as a family of functions from $B$ to the trace class operators in $L(H)$.

It is easy to see that there exists a constant $c_{a}$ depending on $a>0$ such that

$$
\left|A(x)\left[V_{t_{1}}(x)-V_{t_{2}}(x)\right] A(x)\right|_{\mathrm{H}-\mathrm{S} H} \leqq c_{a}\|f\|_{1}\left|t_{1}-t_{2}\right|,
$$

valid for all $t_{1}, t_{2} \geqq a$, for all $f$ in $\mathscr{A}$ and $x$ in $B$. The preceding calculations then imply uniform equicontinuity of $\left\{F_{x, f}: x \in B\right.$ and $\left.\|f\|_{1} \leqq 1\right\}$ where $F_{x, f}(t) \equiv V_{t}(x)$, when considered as a family of functions from $\{t \geqq a>0\}$ to the trace class operators in $L(H)$.

Combining our results for $V, W$ and $X$, we see that the family $\left\{F_{f}:\|f\|_{1} \leqq 1\right\}$, where $F_{f}(t, x) \equiv\left(\hat{m}_{t} f\right)^{\prime \prime}(x)$; is uniformly equicontinuous when considered as a family of functions from $\{t \geqq a>0\} \times B$ to the trace class operators in $L(H)$.

We turn our attention now to $\left(q_{t} f\right)(x)$, for $f$ in $\mathscr{A}$. Equation (4) enables us to write

$$
q_{t} f(x)=\hat{m}_{t} f(x)+\int_{0}^{t}\left(\hat{m}_{t-u} r_{u} f\right)(x) d u .
$$

Properties of $r_{u} f(x)$ are given in Proposition 3 of [7]. Given $0<\delta \leqq T$, there are constants $c_{T}$ and $c_{\delta, T}$ such that, for all $f$ in $\mathscr{A}$,

(r-i) $0<t \leqq T \Rightarrow\left\|r_{t} f\right\|_{1} \leqq c_{T} t^{-1 / 2}\|f\|_{1}$, and

(r-ii) $\delta \leqq t_{1}, t_{2} \leqq T \Rightarrow\left\|r_{t_{1}} f-r_{t_{2}} f\right\|_{\infty} \leqq c_{\delta, T}\left|t_{1}-t_{2}\right| \cdot\|f\|_{1}$.

Since $\left\{q_{t}: t>0\right\}$ forms a strongly continuous contraction semigroup on $\mathscr{A}$, it is immediate that $q_{t} f(x)$ is jointly continuous on $B \times(0, \infty)$. $(r-i)$, combined with the inequalities (10) and (19), yields

$$
\left|\left(\hat{m}_{t-u} r_{u} f\right)^{\prime}(x)\right|_{H} \leqq c_{T}\left(1+(t-u)^{-1 / 2}\right) u^{-1 / 2}\|f\|_{1}
$$

and

$$
\left|\left(\hat{m}_{i-u} r_{u} f\right)^{\prime \prime}(x)\right|_{\operatorname{Tr} H} \leqq c_{T}(t-u)^{-1 / 2} u^{-1 / 2}\|f\|_{1},
$$

for all $x$ in $B$ and $0<u<t \leqq T$. The right sides of (37) and (38) are integrable functions of $u$ on $(0, t)$. This justifies interchanging the order of integration and differentiation in calculating derivatives of $\left(q_{t} f\right)(x)$, and we thus obtain the formulas

$$
\left(q_{t} f\right)^{\prime}(x)=\left(\hat{m}_{t} f\right)^{\prime}(x)+\int_{0}^{t}\left(\hat{m}_{t-u} r_{u} f\right)^{\prime}(x) d u
$$

and

$$
\left(q_{t} f\right)^{\prime \prime}(x)=\left(\hat{m}_{t} f\right)^{\prime \prime}(x)+\int_{0}^{t}\left(\hat{m}_{t-u} r_{u} f\right)^{\prime \prime}(x) d u
$$


The convergence of the time integrals in (39) and (40) is in $H$ norm and $\operatorname{Tr} H$ norm respectively. We see that

$$
\left|\left(q_{t} f\right)^{\prime}(x)\right|_{H} \leqq c_{T}\|f\|_{1}\left(1+t^{-1 / 2}\right)
$$

and

$$
\left|\left(q_{t} f\right)^{\prime \prime}(x)\right|_{\operatorname{Tr} H} \leqq c_{T}\|f\|_{1}\left(1+t^{-1 / 2}\right)
$$

for all $x$ in $H$ and $0<t \leqq T$ (we have incorporated general constants into $c_{T}$ ). Thus we easily verify that

$$
(Q f)^{\prime}(x)=\int_{0}^{\infty} e^{-t}\left(q_{t} f\right)^{\prime}(x) d t
$$

with convergence of the integral in $H$ norm, and

$$
(Q f)^{\prime \prime}(x)=\int_{0}^{\infty} e^{-t}\left(q_{t} f\right)^{\prime \prime}(x) d t
$$

with convergence of the latter integral in $\operatorname{Tr} H$ norm.

Finally, we must establish $(x, t)$-continuity of $\left(q_{t} f\right)^{\prime}(x)$ and of $\left(q_{t} f\right)^{\prime \prime}(x)$ on $B \times(0, \infty)$, as well as uniform $x$-continuity of $(Q f)^{\prime \prime}(x)$ on $B$. We will show that in fact $(q f)^{\prime \prime}(x)$ is uniformly continuous on $B \times[a, b]$ where $0<a<b<\infty$. This will easily imply the desired uniform continuity of $(Q f)^{\prime \prime}(x)$. We will not go through a similar and by now straightforward verification of continuity of $\left(q_{t} f\right)^{\prime}(x)$.

We need only consider the second term on the right side of equation (40). Let $\varepsilon>0$ and $0<a<b<\infty$ be given. Assume $a \leqq t_{1} \leqq t_{2} \leqq b$. Then for $x_{1}$ and $x_{2}$ in $B$ and $f$ in $\mathscr{A}$, we have

$$
\begin{aligned}
\left|\int_{0}^{t_{1}}\left(\hat{m}_{t_{1}-u} r_{u} f\right)^{\prime \prime}\left(x_{1}\right) d u-\int_{0}^{t_{2}}\left(\hat{m}_{t_{2}-u} r_{u} f\right)^{\prime \prime}\left(x_{2}\right) d u\right|_{\mathrm{Tr}} \\
\leqq \\
\quad \int_{0}^{t_{1}}\left|\left(\hat{m}_{t_{1}-u} r_{u} f\right)^{\prime \prime}\left(x_{1}\right)-\left(\hat{m}_{t_{1}-u} r_{u} f\right)^{\prime \prime}\left(x_{2}\right)\right|_{\mathrm{Tr}} d u \\
\quad+\int_{0}^{t_{1}}\left|\left[\left(\hat{m}_{t_{1}-u}-\hat{m}_{t_{2}-u}\right)\left(r_{u} f\right)\right]^{\prime \prime}\left(x_{2}\right)\right|_{\mathrm{Tr}} d u+\int_{t_{1}}^{t_{2}}\left|\left(\hat{m}_{t_{2}-u} r_{u} f\right)^{\prime \prime}\left(x_{2}\right)\right|_{\mathrm{Tr}} d u \\
=(\mathrm{i})+(\mathrm{ii})+(\mathrm{iii}), \text { say. }
\end{aligned}
$$

Using the inequality (38) we have

$$
\text { (iii) } \leqq c_{b}\|f\|_{1} \int_{t_{1}}^{t_{2}}\left(t_{2}-u\right)^{-1 / 2} u^{-1 / 2} d u \leqq c_{a, b}\|f\|_{1}\left(t_{2}-t_{1}\right)^{1 / 2}
$$

Thus we can find a $\delta_{1}>0$ such that $\left|t_{1}-t_{2}\right|<\delta_{1}$ implies (iii) $\leqq\|f\|_{1} \varepsilon / 3$. $\delta_{1}$ is independent of the choice of $x_{2}$. For $0<\lambda_{1}<\lambda_{2}<t_{1}$, we write

$$
\text { (i) } \left.=\left[\int_{0}^{\lambda_{1}}+\int_{\lambda_{1}}^{\lambda_{2}}+\int_{\lambda_{2}}^{t_{1}}\right] \text { [integrand of (i) }\right] \equiv(\text { iv })+(v)+(v i), \text { say. }
$$


We may choose $0<\lambda_{1} \leqq a / 2$ such that

$$
\text { (iv) } \leqq c_{b}\|f\|_{1} \int_{0}^{\lambda_{1}}\left(t_{1}-u\right)^{-1 / 2} u^{-1 / 2} d u \leqq \varepsilon\|f\|_{1} / 6
$$

for all $t_{1}$ in $[a, b]$ and all $x_{1}$ and $x_{2}$ in $B$. If $\lambda_{2} \geqq a$, then

$$
\text { (vi) } \leqq c_{a, b}\|f\|_{1} \int_{\lambda_{2}}^{t_{1}}\left(t_{1}-u\right)^{-1 / 2} d u \text {. }
$$

Choosing $\lambda_{2}$ such that $0<2 c_{b}\left(t_{1}-\lambda_{2}\right)^{1 / 2}=\varepsilon / 6$, we obtain (vi) $\leqq \varepsilon\|f\|_{1} / 6 . \lambda_{2}$ is independent of $x_{1}$ and $x_{2}$ but does depend on $t_{1}$. In the integrand of $(\mathrm{v}), 0<d \equiv\left[\varepsilon / 12 c_{b}\right]^{2}$ $=t_{1}-\lambda_{2} \leqq t_{1}-u \leqq b$. Although $\lambda_{2}$ depends on $t_{1}, d$ does not. Since also in (v), $u$ is bounded away from 0 , we see that a $\delta_{2}>0$ exists such that $\left\|x_{1}-x_{2}\right\|<\delta_{2}$ implies (v) $\leqq \varepsilon\|f\|_{1} / 6 . \delta_{2}$ is independent of $\lambda_{2}$ and of $t_{1}$. Thus for $\left\|x_{1}-x_{2}\right\|<\delta_{2}$ and for all $t_{1}$ in $[a, b]$ we have (i) $\leqq \varepsilon\|f\|_{1} / 3$. We may similarly decompose (ii) into a sum of three integrals, and obtain the existence of a $\delta_{3}>0$ independent of $x_{2}$ such that $\left|t_{1}-t_{2}\right|<\delta_{3}$ implies (ii) $\leqq \varepsilon\|f\|_{1} / 3$. Finally, there exists a $\delta_{4}>0$ such that $\left(x_{i}, t_{i}\right)$ $\in B \times[a, b](i=1,2)$ and $\left\|x_{1}-x_{2}\right\|+\left|t_{1}-t_{2}\right|<\delta_{2}$ implies

$$
\text { (i) }+ \text { (ii) }+(\text { iii }) \leqq \varepsilon\|f\|_{1}
$$

for all $f$ in $\mathscr{A}$. This establishes uniform continuity of $\left(q_{t} f\right)^{\prime \prime}(x)$ on $B \times[a, b]$.

Referring to equation (44) for $(Q f)^{\prime \prime}(x)$, and making a decomposition of the form

$$
\int_{0}^{\infty}=\int_{0}^{a}+\int_{a}^{b}+\int_{b}^{\infty}
$$

we immediately verify uniform continuity of $(Q f)^{\prime \prime}(x)$.

\section{REFERENCES}

1. E. B. Dynkin, Markov processes. Vol. I, Fizmatgiz, Moscow, 1963; English transl., Die Grundlehren der math. Wissenschaften, Band 121, Academic Press, New York, 1965. MR 33 \#1886; \#1887.

2. L. Gross, Measurable functions on Hilbert space, Trans. Amer. Math. Soc. 105 (1962), 372-390. MR 26 \#5121.

3. - Abstract Wiener spaces, Proc. Fifth Berkeley Sympos. Math. Statist. and Probability (Berkeley, Calif., 1965/66), vol. II: Contributions to Probability Theory, part 1, Univ. of California Press, Berkeley, Calif., 1967, pp. 31-42. MR 35 \#3027.

4. —_ Potential theory on Hilbert space, J. Functional Analysis 1 (1967), 123-181. MR 37 \#3331.

5. H. H. Kuo, Stochastic integrals in abstract Wiener space (to appear).

6. H. H. Kuo and M. A. Piech, Stochastic integrals and parabolic equations in abstract Wiener space (to appear).

7. M. A. Piech, A fundamental solution of the parabolic equation on Hilbert space, J. Functional Analysis 3 (1969), 85-114. MR 40 \#4815. 
8. M. A. Piech, Regularity properties for families of measures on a metric space, Proc. Amer. Math. Soc. 24 (1970), 307-311. MR 41 \#5582.

9. —, A fundamental solution of the parabolic equation on Hilbert space. II: The semigroup property, Trans. Amer. Math. Soc. 150 (1970), 257-286.

10. - Some regularity properties of diffusion processes on abstract Wiener space, J. Functional Analysis 8 (1971), 153-172.

11. I. E. Segal, Distributions in Hilbert space and canonical systems of operators, Trans. Amer. Math. Soc. 88 (1958), 12-41. MR 21 \#1545.

Department of Mathematics, State University of New York at Buffalo, Amherst, NEW YoRK 14226 